Mannheimer Manuskripte 232

math.DG/9903105

\title{
COHERENT STATE EMBEDDINGS, POLAR DIVISORS AND CAUCHY FORMULAS
}

\author{
STEFAN BERCEANU AND MARTIN SCHLICHENMAIER
}

\begin{abstract}
A BSTRACT. For arbitrary quantizable compact Kähler manifolds, relations between the geometry given by the coherent states based on the manifold and the algebraic (projective) geometry realised via the coherent state mapping into projective space, are studied. Polar divisors, formulas relating the scalar products of coherent vectors on the manifold with the corresponding scalar products on projective space (Cauchy formulas), two-point, three-point and more generally cyclic $m$-point functions are discussed. The three-point function is related to the shape invariant of geodesic triangles in projective space.
\end{abstract}

\section{INTRODUCTION}

In this article the close relations between the coherent state approach appearing in quantum mechanics and certain aspects of algebraic geometry, respectively Kähler geometry are considered. We analyse the case where the phase-space manifold of the theory is a compact Kähler manifold $(M, \omega)$. The symplectic structure which gives the kinematics of the theory is defined via the Kähler form $\omega$. The geometric quantization condition requires the existence of a line bundle (i.e. the quantum line bundle) with curvature essentially equal to the Kähler form. This implies that the phase-space manifold is projective algebraic. Hence it admits an embedding into projective space. An embedding can be explicitly given by the global sections of a suitable tensor power of the quantum line bundle. It is usually known as Kodaira embedding. Vice versa, every submanifold of projective space is a quantizable Kähler manifold.

Berezin's coherent states [7] in their reformulation and generalization due to Rawnsley [25] define also an embedding into projective space. It turns out that this embedding is nothing else as the Kodaira embedding (respectively its conjugate) with respect to an orthonormal basis of the space of global holomorphic sections of a suitable tensor power of the quantum line bundle. Here the scalar product is induced by the Kähler

Date: 17.3.99.

1991 Mathematics Subject Classification. 58F06; 53C55; 32C17; 14E25; 81R30; 81S10.

Key words and phrases. Coherent states, quantization, Kähler manifolds, shape invariant, Calabi's diastatic function, projective embeddings.

This work was partially supported by the Volkswagen-Stiftung (RiP-program at Oberwolfach). 
form (see Section 2 for details). Having the possible inhomogeneous readership in mind we recall the basics of the afore mentioned concepts in Section 2. In this way we also explain our notation and prove some results used later on.

The main goal of this article is to study relations between the geometry of the quantizable Kähler manifold using coherent states and the algebraic (or projective) geometry of the embedded manifold in $\mathbb{P}^{N}(\mathbb{C})$. Such kind of relations for homogeneous manifolds (with respect to Perelomov's coherent states) were studied by Berceanu [1],[2],[4], [5], [6]. Here we will make similar definitions and prove some analogous results for arbitrary compact Kähler manifolds. Clearly, now we have to use the coherent states of BerezinRawnsley.

The first objects we introduce are the polar divisors (Section 3). The polar divisor of a point on $M$ is the divisor consisting of the points on the manifold whose coherent states are orthogonal to the coherent state associated to the fixed point. It turns out that the polar divisor is indeed a divisor in the sense of algebraic geometry. It should not be confused with the divisor of the polar part of a meromorphic function.

The polar divisors are useful for many purposes. It was shown by Berceanu that for Grassmannians and more general for symmetric spaces [2],[3],[4] the polar divisor $\Sigma_{x}$ (with respect to Perelomov's coherent states) coincides with the cut-locus of the point $x$. For general compact Kähler manifolds the polar divisors describe the zero-sets of two-point functions (and via them also of the $m$-point functions). They appear as singularity sets of the analytic extensions for real-analytic metrics in the bundle and as singularity sets of the covariant two-point Berezin symbols.

Next (Section 4) we discuss "Cauchy formulas". Under a "Cauchy formula" we understand a relation between the scalar product of the coherent states (more precisely, of the coherent vectors) associated to two points on the manifold (again more precisely, associated to points in the total space of the quantum line bundle) and the scalar product of the via the coherent state map embedded two points (more precisely, the scalar product of certain homogeneous representatives of the embedded points). The main results are contained in Theorem 4.5 and the propositions in Section 4. The denomination "Cauchy formula" was used in this context the first time in [1] for Perelomov's coherent states on flag manifolds. For the Grassmannian the appearing formulas are essentially the (Binet-)Cauchy formulas [17, p.10] which give relations between the intrinsic metric on the Grassmannian and the pull-back of the Fubini-Study metric obtained via the Plücker embedding.

In the remaining part of Section 4 the two-point function and cyclic $m$-point functions are discussed. Considered on $M \times M$ the complex-valued two-point function has a phase ambiguity. This ambiguity can either be removed by considering the modulus of the function or alternatively by fixing a holomorphic section of the quantum line bundle as a reference lift to the quantum line bundle. In the first case one ends up with the two-point function studied by Cahen, Gutt and Rawnsley [14] which is related to Calabi's diastatic function. But it turns out that the complex-valued "non-canonical" two-point function plays at least a very useful intermediate rôle. The polar divisors 
appear naturally in this context as zero sets of two-point functions. Next, cyclic $m$ point functions are introduced. They are well-defined on $M^{\times m}$ and invariant under cyclic permutations of its arguments. It is shown that they are invariant under pullback via the coherent state embedding into projective space. They can be expressed in terms of the Cayley distances of the embedded points in projective space and a phase factor, depending on the points. The three-point function is studied in more detail. Here the phase is related to the shape invariant of the geodesic triangle which has the embedded points as vertices. The shape invariant was introduced 1939 by Blaschke and Terheggen [10]. By a result of Hangan and Masala [19] the phase can be calculated via integrating the Kähler form over geodesic triangles. See the closing Theorem 4.8 for the detailed result.

\section{Coherent state embedding}

\subsection{Quantizable Kähler manifolds and Kodaira embedding.}

Let $(M, \omega)$ be a Kähler manifold of complex dimension $n$, i.e. $M$ a complex manifold and $\omega$ a Kähler form on $M$. In the following we will mainly consider compact Kähler manifolds. If nothing else is said we will assume compactness. A further data we need is the triple $(L, h, \nabla)$, with a holomorphic line bundle $L$ on $M$, a hermitian metric $h$ on $L$ (with the convention that it is conjugate linear in the first argument) and a connection $\nabla$ compatible with the metric on $L$ and the complex structure. With respect to local holomorphic coordinates of the manifold and with respect to a local holomorphic frame for the bundle the metric $h$ can be given as

$$
h\left(s_{1}, s_{2}\right)(x)=\hat{h}(x) \overline{\hat{s}_{1}}(x) \hat{s}_{2}(x),
$$

where $\hat{s}_{i}$ is a local representing function for the section $s_{i}(i=1,2)$ and $\hat{h}$ is a locally defined real-valued function on $M$. The compatible connection is uniquely defined and is given in the local coordinates as $\nabla=\partial+(\partial \log \hat{h})+\bar{\partial}$. The curvature of $L$ is defined as the two-form

$$
F(X, Y)=\nabla_{X} \nabla_{Y}-\nabla_{Y} \nabla_{X}-\nabla_{[X, Y]}
$$

where $X$ and $Y$ are vector fields on $M$. In the local coordinates the curvature can be expressed as

$$
F=\bar{\partial} \partial \log \hat{h}=-\partial \bar{\partial} \log \hat{h} .
$$

A Kähler manifold $(M, \omega)$ is called quantizable if there exists such a triple $(L, h, \nabla)$ which obeys

$$
F(X, Y)=-\mathrm{i} \omega(X, Y) .
$$

The condition (2.4) is called the (pre)quantum condition. The bundle $(L, h, \nabla)$ is called a (pre)quantum line bundle. Usually we will drop $\nabla$ and sometimes also $h$ in the notation. 
For the following we assume $(M, \omega)$ to be a quantizable Kähler manifold with quantum line bundle $(L, h, \nabla)$. Let us note some important consequences of the quantum condition. Firstly, we get for the Chern form of the line bundle $L$ the relation

$$
c(L)=\frac{\mathrm{i}}{2 \pi} F=\frac{\omega}{2 \pi} .
$$

This implies that $L$ is a positive line bundle. In the terminology of algebraic geometry it is an ample line bundle. This says that there exists a tensor power $L^{m_{0}}:=L^{\otimes m_{0}}$ with $m_{0}$ a positive integer such that $M$ can be holomorphically embedded into projective space $\mathbb{P}^{N}(\mathbb{C})$ using the global holomorphic sections of $L^{m_{0}}$. Let us describe this embedding in more detail. We will denote the space of global holomorphic sections by $\mathrm{H}^{0}\left(M, L^{m_{0}}\right)$, or depending on the context, also as $\Gamma_{h o l}\left(M, L^{m_{0}}\right)$ (if we regard it as subspace of the space of differentiable sections), resp. by $\mathcal{H}$ (if we regard it as the quantum Hilbert space). By compactness of the manifold $M$ this vector space is finite-dimensional. We take $N=\operatorname{dim} \mathrm{H}^{0}\left(M, L^{m_{0}}\right)-1$ and after fixing a basis of the global sections the embedding is given as

$$
\varphi: M \hookrightarrow \mathbb{P}^{N}(\mathbb{C}), \quad z \mapsto \varphi(z)=\left(s_{0}(z): s_{1}(z): \ldots: s_{N}(z)\right) \in \mathbb{P}^{N}(\mathbb{C}) .
$$

Here we denote the point $\varphi(z)$ in projective space by its homogeneous coordinates. Recall that two sets of homogeneous coordinates correspond to the same point if and only if they are a non-zero scalar multiple of each other. To evaluate the sections one chooses local representing functions for the sections. Clearly, they are only welldefined up to a common scalar function. Hence, only after passing to the projective space the map will be well-defined. The conclusion that from the positivity of the line bundle it follows that there exists such an embedding is the content of Kodaira's embedding theorem, see [18], [30]. By Chow's theorem [18, p.166] compact submanifolds of $\mathbb{P}^{N}(\mathbb{C})$ are projective varieties, i.e. they can be given as zero-sets of a finite number of homogeneous polynomials in the coordinates of $\mathbb{P}^{N}(\mathbb{C})$, see [26], [18]. Hence, we obtain the first part of the following important observation

Observation 2.1. Quantizable compact Kähler manifolds are submanifolds of $\mathbb{P}^{N}(\mathbb{C})$, hence projective algebraic. Conversely, every projective algebraic manifold will be a quantizable Kähler manifold.

The second part will follow from the discussion further down in this section.

In the language of Kähler geometry quantizable compact Kähler manifolds are Hodge manifolds [30], [18]. This is due to the fact that from the relation (2.5) it follows that the class of $\omega$ is $2 \pi$ times a Chern class, hence a integral class, i.e. a class which gives an integer when integrated over a closed 2-surface in $M$.

The number $N$ can be explicitly given with the help of the Grothendieck-HirzebruchRiemann-Roch Theorem [20], [26]. A different choice of basis taken for the embedding corresponds to a holomorphic automorphism of $\mathbb{P}^{N}(\mathbb{C})$, i.e. to an element of $\operatorname{PGL}(N+1, \mathbb{C})$ mapping the images onto each other. 
In the following we will assume that $L$ is already very ample. This says that $M$ can already be embedded using the global sections of $L$. If this is not yet the case we can always choose a $m_{0} \in \mathbb{N}$ such that $L^{m_{0}}$ is very ample. Now in generality for a $m^{\text {th }}$ tensor power $L^{m}$ of the bundle $L$ a metric $h^{(m)}$ and a connection $\nabla^{(m)}$ is given by

$$
\begin{gathered}
h^{(m)}:=\underbrace{h \otimes \cdots \otimes h}_{m \text { times }}, \\
\nabla^{(m)}=\nabla \otimes 1 \otimes \cdots \otimes 1+1 \otimes \nabla \otimes \cdots \otimes 1+\cdots+1 \otimes \cdots \otimes 1 \otimes \nabla .
\end{gathered}
$$

The corresponding local objects are (if one takes the $m^{\text {th }}$ tensor power of the frame of $L$ as frame for $L^{m}$ )

$$
\widehat{h^{(m)}}=(\hat{h})^{m}, \quad \nabla^{(m)}=\partial+m(\partial \log \hat{h})+\bar{\partial}, \quad F^{(m)}=m F=-\mathrm{i} m \omega .
$$

Hence, for every $m \in \mathbb{N}$ the bundle $\left(L^{m}, h^{(m)}, \nabla^{(m)}\right)$ is a quantum line bundle for the Kähler manifold $(M, m \omega)$. Note that the underlying complex manifold remains the same, only the Kähler form is multiplied by an integer ${ }^{1}$. So, if we start with $\left(M, m_{0} \omega\right)$ the corresponding quantum line bundle $L^{m_{0}}$ is very ample.

A second consequence of the quantum condition (2.4) is that the metric in the quantum bundle can be expressed with the help of a local Kähler potential [16]. For a Kähler manifold there exist locally real-valued (non-unique) functions $K$ such that $\omega=\mathrm{i} \partial \bar{\partial} K$. With the quantum condition (2.4) it follows from (2.3)

$$
\omega=\mathrm{i} \bar{\partial} \partial \log \hat{h} \text {. }
$$

Hence a local Kähler potential can be given as

$$
K(z)=-\log \hat{h}(z), \quad \text { resp. }, \quad \hat{h}(z)=\exp (-K(z)) .
$$

Recall the Kähler structure of the projective space $\mathbb{P}^{N}(\mathbb{C})$. The points $[z]$ in $\mathbb{P}^{N}(\mathbb{C})$ are given by their homogeneous coordinates $[z]:=\left(z_{0}: z_{1}: \ldots: z_{N}\right)$. In the affine chart $V_{0}$ consisting of the points with $z_{0} \neq 0$ we take $w_{j}=z_{j} / z_{0}$ with $j=1, \ldots, N$ as holomorphic coordinates. In the similar way we define affine charts $V_{k}, k=1, \ldots, N$ and corresponding holomorphic coordinates. The union $\bigcup_{k=0}^{N} V_{k}$ is now an affine covering of $\mathbb{P}^{N}(\mathbb{C})$. Denote by

$$
\tau: \mathbb{C}^{N+1}-\{0\} \quad \rightarrow \quad \mathbb{P}^{N}(\mathbb{C})
$$

the projection which is obtained by identifying the whole line through 0 and the point $\mathrm{z}$ with the point in projective space with homogeneous coordinates $[z]$. The Kähler form

\footnotetext{
${ }^{1}$ The process that starting from one line bundle $L$ one obtains for every $m \in \mathbb{N}$ a quantization allows to introduce semi-classical limits of the quantization scheme (geometric quantization, Berezin-Toeplitz quantization, coherent state quantization,..), to prove approximation results for them (e.g. see [11]), and to show the existence of star products [13],[14],[27], [29].
} 
on $\mathbb{P}^{N}(\mathbb{C})$ is the Fubini-Study fundamental form. On $V_{0}$ it is given as

$$
\omega_{F S}:=\mathrm{i} \frac{\left(1+\|w\|^{2}\right) \sum_{i=1}^{N} d w_{i} \wedge d \bar{w}_{i}-\sum_{i, j=1}^{N} \bar{w}_{i} w_{j} d w_{i} \wedge d \bar{w}_{j}}{\left(1+\|w\|^{2}\right)^{2}} .
$$

Here $\|w\|^{2}:=\sum_{i=1}^{N} \bar{w}_{i} w_{i}$, as usual. Alternatively it can be described as [21]

$$
\tau^{*} \omega_{F S}(z)=\mathrm{i} \bar{\partial} \partial \log \|z\|^{2} .
$$

Over $\mathbb{P}^{N}(\mathbb{C})$ we have the tautological line bundle $U$. Its fiber over $[z]$ consists of the line through 0 and $z$. Taking the standard metric in $\mathbb{C}^{N+1}$ it is endowed with a natural hermitian fiber metric. Note that the manifold $\mathbb{C}^{N+1}-\{0\}$ can be identified with the total space of $U$ with the zero section removed. With respect to the affine chart $V_{0} \cong \mathbb{C}^{N}$ we can write two elements of the same fiber over $w \in \mathbb{C}^{N}$ as

$$
s_{1}=\alpha \cdot\left(1, w_{1}, \ldots, w_{N}\right), \quad s_{2}=\beta \cdot\left(1, w_{1}, \ldots, w_{N}\right),
$$

and obtain

$$
\bar{s}_{1} \cdot s_{2}=\bar{\alpha} \cdot \beta \cdot\left(1+\|w\|^{2}\right) .
$$

Hence, the local representing function in $V_{0}$ for the hermitian metric of the line bundle $U$ (and with respect to the standard frame $V_{0} \rightarrow \mathbb{C} \times V_{0}, w \mapsto(1, w)$ ) is

$$
\hat{k}(w)=1+\|w\|^{2} .
$$

The quantum line bundle is the dual of the tautological bundle, the hyperplane bundle $H=U^{*}$. The hermitian metric of the hyperplane bundle can be given in the affine chart by the representing function

$$
\hat{h}(w)=\frac{1}{1+\|w\|^{2}} .
$$

The global holomorphic sections of $H$ can be identified with the linear forms in the $N+1$ coordinate functions $Z_{i}$.

We were using the term "the quantum line bundle" indicating that there is up to algebraic isomorphy just one line bundle with curvature form $-\mathrm{i} \omega$. In general this is not the case. But for the projective space there is for every degree up to isomorphy just one line bundle and the degree is fixed by the curvature. Hence for the projective space (with the Fubini-Study Kähler form) the quantum line bundle is fixed. In fact the same is true for any simply-connected, compact, quantizable Kähler manifold. In this case there is at most one line bundle which has a given candidate as curvature form, see [22, Thm. 2.2.1]. Here a warning is in order. It is not excluded that for the same underlying complex manifold there exist (essentially) different Kähler forms and hence essentially different associated quantum line bundles.

If $M$ is a projective submanifold of $\mathbb{P}^{N}(\mathbb{C})$ with

$$
i: M \hookrightarrow \mathbb{P}^{N}(\mathbb{C})
$$


the inclusion then $\left(M, i^{*} \omega_{F S}\right)$ is a Kähler manifold [18], [30] which is quantizable with the associated quantum line bundle $\left(i^{*} H, i^{*} h\right)$. Here $i^{*}$ is nothing else as the restriction to the submanifold. Hence, projective manifolds are quantizable Kähler manifolds. This shows the second statement in Observation 2.1.

We have to stress an important fact. If $\left(M, \omega_{M}\right)$ is a quantizable Kähler manifold with very ample quantum line bundle $L$ then we saw that $L$ induces an embedding $i: M \hookrightarrow$ $\mathbb{P}^{N}(\mathbb{C})$. By the construction $i^{*} H \cong L$ as holomorphic line bundle. Now $\left(M, i^{*} \omega_{F S}\right)$ is a Kähler manifold with the same underlying complex manifold structure. But in general $\omega_{M} \neq i^{*} \omega_{F S}$, so the Kähler structure of $M$ does not coincide with the induced Kähler structure coming from the embedding. The embedding is in general not an isometric (Kähler) embedding. The situation is very much related to Calabi's diastatic function, [15], [13]. In general we only know the identity of the deRham classes $\left[\omega_{M}\right]=\left[i^{*} \omega_{F S}\right]$. This follows from the identity of the Chern classes $c_{1}(L)=c_{1}\left(i^{*} H\right)=i^{*} c_{1}(H)$ and from the fact that by the quantum condition the Kähler forms represent (up to a factor) the curvature class.

In the compact case the Kähler form $\omega$ (hence the metric on $M$ ) fixes via the quantum condition the hermitian bundle metric in $L$ up to a scalar constant:

Proposition 2.2. Let $(M, \omega)$ be a quantizable compact Kähler manifold with quantum bundles $(L, h)$ and $\left(L, h^{\prime}\right)$ then $h=\mathrm{e}^{\alpha} \cdot h^{\prime}$, with $\alpha \in \mathbb{R}$.

Proof. Represent the metric $h$ and $h^{\prime}$ with respect to a local frame of the (same) bundle $L$ as local functions $\hat{h}$ and $\hat{h}^{\prime}$. By the quantum condition (2.4) we obtain for the Kähler form

$$
\omega=\mathrm{i} \partial \bar{\partial} \log \hat{h}=\mathrm{i} \partial \bar{\partial} \log \hat{h}^{\prime}
$$

Hence $\partial \bar{\partial}\left(\log \hat{h}-\log \hat{h}^{\prime}\right)=0$ or equivalently $\log \left(\hat{h} / \hat{h}^{\prime}\right)$ is a locally defined harmonic function. But the quotient of the two metrics is a globally defined function. Hence $\log \left(\hat{h} / \hat{h}^{\prime}\right)$ is a globally defined harmonic function on the compact manifold and hence a constant $\alpha \in \mathbb{R}$. This shows the claim.

\subsection{Embedding via coherent states.}

We now want to describe an (anti-)holomorphic embedding of the Kähler manifold $(M, \omega)$ into projective space using coherent states. We use Berezin's coherent states [7], [8], [9] in the coordinate independent global version due to Rawnsley, see [25], [13].

First we have to introduce a scalar product in the space of global holomorphic sections of the quantum line bundle $L$. With the normalized volume form

$$
\Omega:=(-1)^{\left(\begin{array}{l}
n \\
2
\end{array}\right)} \frac{1}{n !} \underbrace{\omega \wedge \ldots \wedge \omega}_{n \text { times }}
$$


and with the fiber metric $h$ we can introduce a scalar product and a norm on the space of differentiable sections $\Gamma_{\infty}(M, L)$

$$
\langle\varphi, \psi\rangle:=\int_{M} h(\varphi, \psi) \Omega, \quad\|\varphi\|:=\sqrt{\langle\varphi, \varphi\rangle} .
$$

Using local representing functions $\hat{\varphi}$ and $\hat{\psi}$ for the sections and $\hat{h}$ for the metric the scalar product can be described as

$$
\langle\varphi, \psi\rangle=\int_{M} \hat{h}(z) \overline{\hat{\varphi}(z)} \hat{\psi}(z) \Omega(z)=\int_{M} \exp (-K(z)) \overline{\hat{\varphi}(z)} \hat{\psi}(z) \Omega(z) .
$$

In the second form we used the local Kähler potential (2.10). Clearly, these integrals should be calculated locally and their values patched together by a partition of unity argument ${ }^{2}$.

The scalar product can be restricted to the finite dimensional subspace of global holomorphic sections.

Recall that we assume the quantum line bundle to be already very ample. Denote by $\pi: L \rightarrow M$ the bundle projection and by $L_{0}$ the total space of $L$ with the zero section $0(M)$ removed. Fix $q \in L_{0}$ and take an arbitrary holomorphic section $s$ of $L$. By evaluation of the section at $x=\pi(q)$ the relation

$$
s(\pi(q))=\hat{q}(s) \cdot q
$$

defines a linear form

$$
\hat{q}: \Gamma_{h o l}(M, L) \rightarrow \mathbb{C}, \quad s \mapsto \hat{q}(s) .
$$

Using the scalar product on the space of global sections, by Riesz's theorem there exists exactly one holomorphic section $e_{q}$ with

$$
\left\langle e_{q}, s\right\rangle=\hat{q}(s), \quad \text { for all } \quad s \in \Gamma_{h o l}(M, L) .
$$

If we choose an orthonormal basis $s_{j}, j=0, \ldots, N:=\operatorname{dim} \Gamma_{h o l}(M, L)-1$ then $e_{q}$ can be explicitly given as

$$
e_{q}=\sum_{j=0}^{N} \overline{\hat{q}\left(s_{j}\right)} s_{j} .
$$

Let $x=\pi(q)$ and choose $q^{\prime} \in \pi^{-1}(x)$ with $q^{\prime} \neq 0$ then there is a $c \in \mathbb{C}^{*}$ with $q^{\prime}=c q$. From (2.23) we conclude $\widehat{q^{\prime}}=c^{-1} \widehat{q}$ and using (2.25) we obtain

$$
e_{c q}=\bar{c}^{-1} \cdot e_{q} .
$$

\footnotetext{
${ }^{2}$ Sometimes it is useful to write $\hat{h}(z, \bar{z})$, resp. $K(z, \bar{z})$ to remind of the fact that these functions are not holomorphic in $z$ and (even more important) to consider the possibility to extend the objects analytically to $h(z, \bar{w})$, resp. $K(z, \bar{w})$.
} 
We obtain two mappings

$$
\begin{array}{rlrl}
L_{0} \rightarrow \Gamma_{h o l}(M, L)^{*}, & q & \mapsto \hat{q}, & \text { and } \\
L_{0} \rightarrow \Gamma_{h o l}(M, L), & q \mapsto e_{q} . &
\end{array}
$$

The first one is holomorphic, the second one antiholomorphic. By the above relations both maps are well-defined on $M$ if we pass to the projectivized vector spaces

$$
\begin{array}{rlrl}
M \rightarrow \mathbb{P}\left(\Gamma_{h o l}(M, L)^{*}\right), & & \left.x \mapsto \widehat{\pi^{-1}(x)}\right], & \text { and } \\
M \rightarrow \mathbb{P}\left(\Gamma_{h o l}(M, L)\right), & x \mapsto\left[e_{\pi^{-1}(x)}\right] . &
\end{array}
$$

Here $[v]$ denotes the equivalence class of a vector $v$ of a vector space $V$ in the projectivized vector space $\mathbb{P}(V)$. In abuse of notation we understand by $\pi^{-1}(x)$ only the non-zero elements of the fiber over $x$.

Note that $\hat{q} \equiv 0$ or equivalently $e_{q} \equiv 0$ would imply that all sections $s \in \Gamma_{\text {hol }}(M, L)$ will vanish at $\pi(q)$ and this contradicts the very ampleness of $L$.

Depending on $q \in L_{0}$ the sections $e_{q} \in \Gamma_{\text {hol }}(M, L)$ are called coherent vectors. Depending on $x \in M$ the $\left[e_{\pi^{-1}(x)}\right] \in \mathbb{P}\left(\Gamma_{\text {hol }}(M, L)\right)$ are called coherent states. To simplify the notation we will set $e_{x}:=\left[e_{\pi^{-1}(x)}\right]$. The mappings (2.29) and (2.31) are the coherent vector mapping, resp. the coherent state mapping.

To identify $\mathbb{P}\left(\Gamma_{h o l}(M, L)\right)$ with $\mathbb{P}^{N}(\mathbb{C})$ we choose an orthonormal basis. The description (2.26) shows that the coherent state mapping is given as

$$
x \mapsto e_{x}=\left[e_{\pi^{-1}}(x)\right] \mapsto\left(\overline{\hat{q}\left(s_{0}\right)}: \overline{\hat{q}\left(s_{1}\right)}: \ldots: \overline{\hat{q}\left(s_{N}\right)}\right)=\left(\overline{s_{0}(x)}: \overline{s_{1}(x)}: \ldots: \overline{s_{N}(x)}\right),
$$

For the last equality we used $s_{j}(x)=s_{j}(\pi(q))=\hat{q}\left(s_{j}\right) \cdot q$.

Proposition 2.3. The map (2.32)

$$
M \rightarrow \mathbb{P}\left(\Gamma_{\text {hol }}(M, L)\right) \cong \mathbb{P}^{N}(\mathbb{C}),
$$

is an antiholomorphic embedding. Up to complex conjugation it coincides with the Kodaira embedding (2.6) obtained with respect to the chosen orthonormal basis.

Proof. That the map is well-defined we showed above. That it is an embedding follows from the observation that Equation (2.32) is up to complex conjugation nothing else as the Kodaira embedding with respect to the very ample line bundle $L$.

In the following it will be more convenient to consider the complex conjugate of the coherent state embedding (2.32)

$$
x \mapsto \overline{e_{x}}=\left[\overline{e_{\pi^{-1}}(x)}\right] \mapsto \quad\left(\hat{q}\left(s_{0}\right): \hat{q}\left(s_{1}\right): \ldots: \hat{q}\left(s_{N}\right)\right)=\left(s_{0}(x): s_{1}(x): \ldots: s_{N}(x)\right),
$$

which is a holomorphic embedding. We will use the term coherent state embedding also for (2.34) if there is no danger of confusion. 
Note that a different orthonormal basis (ONB) will yield an embedding which is equivalent under a $\mathrm{PU}(N+1)$ action to the chosen one.

In the language of physics Proposition 2.3 means that the phase space of a mechanical system (assumed here to be Kählerian) can be embedded via coherent states into a projectivized Hilbert space, the quantum Hilbert space.

It should be pointed out that the coherent state embedding is not just Kodaira embedding. It is Kodaira embedding using orthonormal sections. The scalar product used to define the orthonormality on $\Gamma_{\text {hol }}(M, L)$ (which should be interpreted as the quantum Hilbert space $\mathcal{H}$ ) is induced by the Kähler form on the manifold and by the hermitian metric in the bundle. In view of the quantization condition the latter itself can be related to the Kähler form of the manifold, see (2.9) and Proposition 2.2. The Kähler form (interpreted as symplectic form) is an important ingredients to the description of the system to be quantized.

If one considers non-compact Kähler manifolds then the scalar product (2.21) on $\Gamma_{\infty}(M, L)$ or more precisely on $\mathrm{L}^{2}(M, L)$ is the starting point. The space $\Gamma_{h o l}(M, L)$ has to be replaced by the subspace $\Gamma_{h o l}^{b}(M, L)$ of bounded holomorphic sections. An orthonormal basis of the subspace defines a map

$$
M \rightarrow \mathbb{P}\left(\Gamma_{h o l}^{b}(M, L)\right) .
$$

This defines an embedding into the infinite dimensional projective space. By the continuity of the evaluation functional (2.23) Riesz's theorem can also be applied to define the coherent vectors. For more details see [25], [31], [23], [24].

We need also the coherent projectors used by Rawnsley

$$
P_{\pi(q)}=\frac{\left|e_{q}\right\rangle\left\langle e_{q}\right|}{\left\langle e_{q}, e_{q}\right\rangle} .
$$

Here we used the convenient bra-ket notation. For $s, t \in \Gamma_{\text {hol }}(M, L)$ the symbol $|s\rangle\langle t|$ denotes the following rank 1 operator of $\Gamma_{h o l}(M, L)\left(\operatorname{resp}\right.$. of $\Gamma_{\infty}(M, L)$ )

$$
|s\rangle\langle t|: r \rightarrow\langle t, r\rangle \cdot s
$$

By the normalization the projectors are indeed only depending on the points $\pi(q)$ of the manifold.

Rawnsley introduced the Epsilon function

$$
\epsilon(\pi(q)):=|q|^{2}\left\langle e_{q}, e_{q}\right\rangle, \quad \text { with } \quad|q|^{2}:=h(\pi(q))(q, q) .
$$

Let $s_{1}$ and $s_{2}$ be two sections. At a fixed point $x=\pi(q)$ we can write $s_{1}(x)=\hat{q}\left(s_{1}\right) q$ and $s_{2}(x)=\hat{q}\left(s_{2}\right) q$ and hence using $(2.25)$

$$
h\left(s_{1}, s_{2}\right)(x)=\overline{\hat{q}\left(s_{1}\right)} \cdot \hat{q}\left(s_{2}\right) \cdot|q|^{2}=\left\langle s_{1}, e_{q}\right\rangle\left\langle e_{q}, s_{2}\right\rangle|q|^{2}=\left\langle s_{1}, P_{x} s_{2}\right\rangle \cdot \epsilon(x) .
$$

After integration we obtain the over-completeness property of the coherent states

$$
\left\langle s_{1}, s_{2}\right\rangle=\int_{M}\left\langle s_{1}, P_{x} s_{2}\right\rangle \epsilon(x) \Omega(x) .
$$


We calculate

$$
\epsilon(x)=|q|^{2}\left\langle e_{q}, e_{q}\right\rangle=|q|^{2} \sum_{j=0}^{N}\left|\hat{q}\left(s_{j}\right)\right|^{2}=\sum_{j=0}^{N}\left|\hat{q}\left(s_{j}\right)\right|^{2} h(x)(q, q)=\sum_{j=0}^{N} h\left(s_{j}, s_{j}\right)(x) .
$$

It was shown in [13, Equ. (3.4)] that for $\epsilon$. const one obtains

$$
\epsilon=\frac{\operatorname{dim} \Gamma_{h o l}(M, L)}{\operatorname{vol}(M)}
$$

On homogeneous Kähler manifolds with a homogeneous quantum line bundle (in particular also with homogeneous metric) the function $\epsilon(x)$ is invariant under moving the point, hence it is a constant. In particular, it is constant for the projective space $\mathbb{P}^{N}(\mathbb{C})$. See Proposition 4.1 for more information.

To compare this approach with the local description used by Berezin we have to choose a section $s_{0} \in \Gamma_{\text {hol }}(M, L), s_{0} \not \equiv 0$. Let $V=\left\{x \in M \mid s_{0}(x) \neq 0\right\}$ be the open subset on which the section does not vanishes ${ }^{3}$. Now $s_{0}$ is a holomorphic frame for the bundle $L$ over $V$. This says that over $V$ every holomorphic (differentiable) section can be described as $s(x)=\hat{s}(x) s_{0}(x)$ with a holomorphic (resp. differentiable) function $\hat{s}$. The mapping $s \mapsto \hat{s}$ defines an isometry of $\Gamma_{h o l}(M, L)$ (resp. of $\Gamma_{\infty}(M, L)$ ) into the $\mathrm{L}^{2}$ space of holomorphic (resp. differentiable) functions on $V$ with respect to the measure $\mu_{s_{0}}(x)=h\left(s_{0}, s_{0}\right)(x) \Omega(x)$.

With respect to the frame $s_{0}$ the function $\hat{h}$ describing the metric is given as $\hat{h}(x)=$ $h\left(s_{0}, s_{0}\right)(x)$. Hence we can describe the scalar product for $\varphi, \psi \in \Gamma_{\infty}(M, L)$ as

$$
\langle\varphi, \psi\rangle=\int_{V} \overline{\hat{\varphi}(x)} \hat{\psi}(x) h\left(s_{0}, s_{0}\right)(x) \Omega(x) .
$$

If we introduced the local Kähler potential given by (2.10). then this can be rewritten as

$$
\langle\varphi, \psi\rangle=\int_{V} \overline{\hat{\varphi}(x)} \hat{\psi}(x) \exp (-K(x)) \Omega(x) .
$$

It is enough to calculate the integral on $V$, because $M \backslash V$ is of (complex) codimension 1 , hence of measure zero, see Section 3. Such a description is always possible. For doing explicit calculations Berezin considered special cases where $V$ is either $\mathbb{C}^{n}$, or a subset of special type of $\mathbb{C}^{n}$ (e.g. bounded symmetric domains) [7], [8], [9].

\section{THE POLAR DIVISOR}

\subsection{The definition of the polar divisor.}

Definition 3.1. Let $x \in M$ and $0 \neq q \in \pi^{-1}(x)$ then the polar divisor $\Sigma_{x}$ associated to $x \in M$ is defined as

$$
\Sigma_{x}:=\left\{x^{\prime} \in M \mid\left\langle e_{q}, e_{q^{\prime}}\right\rangle=0 \quad \text { for } 0 \neq q^{\prime} \in \pi^{-1}\left(x^{\prime}\right)\right\} .
$$

\footnotetext{
${ }^{3}$ In the terminology of Section 3 we remove the support of the divisor of the section $s_{0}$.
} 
I.e. the polar divisor $\Sigma_{x}$ is the set of points on the manifold for which the associated coherent vectors are orthogonal to the coherent vectors associated to $x$.

Due to the relation (2.27) the definition is independent of the representing elements $q$ and $q^{\prime}$. In the context of Perelomov's coherent states the notion of polar divisors was introduced by Berceanu [1],[2],[4],[6]. On the purely geometric side the polar divisor was used earlier by H.H. Wu [32] for the complex Grassmannians. As we will see in the following it has a meaning in much more general situations. There should be no danger of confusion with the notion of polar divisor in complex analysis as the divisor of the polar part of a meromorphic function. (See the remark after Equation (4.7) for a connection.)

Note that for every (meromorphic or holomorphic) section of a line bundle there is an associated divisor in the sense of algebraic geometry. For a thorough treatment of the relation between divisors, line bundles and sections of line bundles see [18, p.130ff], [26]. What we need here are only the following facts. For a holomorphic section $s \not \equiv 0$ of a line bundle the zero-set of the section can be decomposed into a union of (complex) one-codimensional "irreducible subvarieties" which are not necessarily smooth. The complement of the zero-set is an open dense subset of $M^{4}$. Each codimension one irreducible subvariety can be given locally as zero-set of an algebraic function. By the irreducibility the vanishing order along the subvariety is constant. Hence we can assign to the section $s$ the formal sum $(s)$ of (irreducible) codimension one subvarieties with integer coefficients

$$
(s):=\sum_{\substack{Y \text { irreducible } \\ \text { subvarity of } M \\ \text { of codimension } 1}} n_{Y} Y,
$$

where $n_{Y}$ denotes the vanishing order along $Y$. By the compactness of $M$ the sum (3.2) will always be finite. Every such formal sum with $n_{Y} \in \mathbb{Z}$ fulfilling the restriction that $n_{Y} \neq 0$ only for finitely many $Y$ is called a divisor of $M$. The sum $(s)$ is called the divisor of the section s. For meromorphic sections negative integers (corresponding to algebraic poles) are allowed. Two divisors are called linearly equivalent if their difference (as formal sum) is the divisor of a meromorphic function on $M$. Note that the functions are the (meromorphic) sections of the trivial line bundle. By this an equivalence relation is defined. The linear equivalence class of a divisor is called a divisor class. The set of divisor classes carries a natural structure of an abelian group under addition of divisors. For smooth projective varieties (as $M$ is one by the quantization condition) this divisor class group is isomorphic to the group of isomorphy classes of algebraic line bundles, where for the latter the group structure is defined by the tensor product of line bundles. The isomorphism is given by assigning to the line bundle the divisor class of any nontrivial meromorphic section. Note that the divisors of two meromorphic sections of the same line bundle are linearly equivalent.

\footnotetext{
${ }^{4}$ We assume $M$ to be connected and compact
} 
Recall that the coherent vector $e_{q}$ is a section of the quantum line bundle and as introduced above its divisor is given by $\left(e_{q}\right)$. The zero-set of $e_{q}$ (forgetting the multiplicities) is called the the reduced support red $\left(e_{q}\right)$ of the divisor $\left(e_{q}\right)$.

Proposition 3.2. The polar divisor associated to $x$ is the reduced support of a divisor. More precisely,

$$
\Sigma_{x}=\operatorname{red}\left(e_{q}\right), \text { with } q \in \pi^{-1}(x), q \neq 0 .
$$

Proof. Let $q^{\prime} \in L_{0}$, resp. $x^{\prime} \in M, \pi\left(q^{\prime}\right)=x^{\prime}$. From (2.25) it follows $\left\langle e_{q^{\prime}}, e_{q}\right\rangle=\widehat{q^{\prime}}\left(e_{q}\right)$ and

$$
e_{q}\left(x^{\prime}\right)=e_{q}\left(\pi\left(q^{\prime}\right)\right)=\widehat{q^{\prime}}\left(e_{q}\right) \cdot q^{\prime}=\left\langle e_{q^{\prime}}, e_{q}\right\rangle \cdot q^{\prime} .
$$

Hence, $x^{\prime}=\pi\left(q^{\prime}\right)$ is a zero of the section $e_{q}$ if and only if $\left\langle e_{q^{\prime}}, e_{q}\right\rangle=0$. This shows the claim.

By the above proof we see that the multiplicity structure of the zeros of $e_{q}$ and that of $\left\langle e_{q^{\prime}}, e_{q}\right\rangle=0$ are the same. Hence we can indeed consider $\Sigma_{x}$ as a divisor if we assign to it the corresponding multiplicity of its components. Note that due to relation $(2.27)$

$$
\left(e_{q}\right)=\left(e_{q^{\prime}}\right) \text { for } \quad q, q^{\prime} \in \pi^{-1}(x) \backslash\{0\}, \quad x \in M .
$$

Hence we can assign for every $x \in M$ the divisor

$$
\left(e_{x}\right):=\left(e_{\pi^{-1}(x)}\right) \text {, }
$$

to the coherent state $e_{x}$.

We obtain

Corollary 3.3. The polar divisor $\Sigma_{x}$ associated to $x$ is the divisor $\left(e_{x}\right)$ in the sense of algebraic geometry (3.6) of the coherent state $e_{x}$ associated to $x$.

Let $V$ be an open non-empty subset over which the bundle $L$ can be (holomorphically) trivialized, i.e. $L_{\mid V} \cong V \times \mathbb{C}$. We take $q^{\prime}$ above $x^{\prime} \in V$ as $x^{\prime} \mapsto\left(x^{\prime}, 1\right)$ (i.e. we take as $q^{\prime}$ the value at $x^{\prime}$ of the frame given by the trivialization) then using (3.4) we obtain

Proposition 3.4. The function $\hat{e}_{q}\left(x^{\prime}\right):=\left\langle e_{\left(x^{\prime}, 1\right)}, e_{q}\right\rangle$ is the holomorphic local representing function for the section $e_{q}$.

Let $s$ be a global holomorphic section not identically zero. The complement of the divisor $(s)$

$$
V_{s}:=V \backslash(s)=\{x \in M \mid s(x) \neq 0\}
$$

will be an open dense subset of $M$. If we apply Proposition 3.4 to the trivialization obtained by taking as frame the holomorphic section $s$ on $V_{s}$ then $\left(x^{\prime}, 1\right) \cong s\left(x^{\prime}\right)$ and we can reformulate Proposition 3.4 as 
Corollary 3.5. Let $s \not \equiv 0$ be a global holomorphic section. Then with respect to the frame given by the section s a local representing function over $V_{s}$ for the coherent vector $e_{q}$ is given by

$$
\hat{e}_{q}\left(x^{\prime}\right)=\left\langle e_{s\left(x^{\prime}\right)}, e_{q}\right\rangle .
$$

Immediately from the definition of polar divisors we get

Proposition 3.6. The polar divisors obey the symmetry relation

$$
y \in \Sigma_{x} \longleftrightarrow x \in \Sigma_{y} .
$$

Remark. By the definition (2.23) of $\hat{q}$, resp. of $e_{q}(2.25)$ one concludes that $\left\langle e_{q^{\prime}}, e_{q}\right\rangle$ varies antiholomorphically in $q$ and holomorphically in $q^{\prime}$. Hence $q \mapsto \Sigma_{q}$ defines an antiholomorphic family of divisors on $M$ and $q \mapsto \hat{e}_{q}$ an antiholomorphic family of sections for the bundle $L$.

Remark. By Bertini's theorem [20] the divisor of a generic global holomorphic section of the bundle $L$ is a smooth hypersurface. The divisors $\left(e_{q}\right)$ for the coherent vectors are not necessarily generic, so one can not expect them to be smooth in general. See [3] for an example.

Example. Let us consider the simplest example, the projective line $\mathbb{P}^{1}(\mathbb{C})$, resp. the sphere $S^{2}$ with the Kähler structure given by $m(m \in \mathbb{N})$ times the Fubini-Study form

$$
\omega=\frac{\mathrm{i}}{(1+z \bar{z})^{2}} d z \wedge d \bar{z}
$$

as Kähler form with respect to the quasi-global coordinate $z$. The corresponding quantum line bundle is $H^{\otimes m}$, where $H$ is the hyperplane bundle. The coherent vectors in the standard affine chart are given as

$$
e_{\psi(w)}(z)=\frac{m+1}{2 \pi}(1+\bar{w} z)^{m} \psi(z),
$$

where we take the (on this chart) non-vanishing section $\psi$ as reference section. The divisors are formal sums of points with integer coefficients. We denote the divisor corresponding to the point with the coordinate $z_{0}$ by $\left\langle z_{0}\right\rangle$. The polar divisors are calculated (using Corollary 3.3) directly as the zero set of the section (3.11), hence

$$
\Sigma_{w}=m\left\langle-\frac{1}{\bar{w}}\right\rangle, \quad w \neq 0, \infty, \quad \Sigma_{0}=m\langle\infty\rangle, \quad \Sigma_{\infty}=m\langle 0\rangle .
$$

Using the original Definition 3.1 we can also calculate (compare (3.8))

$$
\left\langle e_{\psi\left(w^{\prime}\right)}, e_{\psi(w)}\right\rangle_{H \otimes m}=\frac{m+1}{2 \pi}\left(1+\bar{w} w^{\prime}\right)^{m},
$$

yielding (in accordance with Corollary 3.3) clearly the same set of points where (3.13) vanishes.

In particular for $m>1$ the divisors appearing as divisors of coherent states are not smooth because they have higher multiplicities. 
The polar divisors appear at many places. It was shown by Berceanu that for Grassmannians and more general for Hermitian symmetric spaces [2],[3],[4] the polar divisor $\Sigma_{x}$ (with respect to Perelomov's coherent states) coincides with the cut-locus of the point $x$. Recall that for a geodesic starting at $x$ the cut-point $y$ is the point where the geodesic ceases to be the shortest curve connecting $x$ and $y^{\prime}$ with $y^{\prime}>y$ on the geodesic. The cut-locus consists of all cut-points. For more details see the above references.

As we will see in the following section for arbitrary compact Kähler manifolds the polar divisors describe the zero-sets of two-point functions and more general of $m$-point functions. They appear as singularity sets of the analytic extensions of real-analytic metrics in the bundle (see (4.8)) and as singularity sets of the covariant two-point Berezin symbols.

\section{Cauchy formulas and multi-point functions}

\subsection{Coherent projective Kähler embedding.}

Let the compact Kähler manifold $\left(M, \omega_{M}\right)$ be embedded via the (holomorphic) coherent state map (2.34) with respect to the very ample line bundle $\left(L, h_{L}\right): \quad i: M \hookrightarrow$ $\mathbb{P}^{N}(\mathbb{C})$. Fix the orthonormal sections $s_{j}, j=0, \ldots, N$ of the quantum bundle $L$. Let $t_{j}, j=0, \ldots, N$ be the sections of the hyperplane bundle $H$ over $\mathbb{P}^{N}(\mathbb{C})$ corresponding to the linear forms $Z_{j}, j=0, \ldots, N$. By construction we have $i^{*} H \cong L$ and $i^{*}\left(t_{j}\right)=s_{j}$ (i.e. $s_{j}(x)=t_{j}(i(x))$ under the identification of the bundles. It is well-known that the $t_{j}$ are orthogonal sections of $H$ with norm independent of $j$. We will denote the rescaled orthonormal section by $t_{j}^{\prime}$ and obtain $s_{j}=\tau i^{*}\left(t_{j}^{\prime}\right)$ with a factor $\tau$ independent of $j$. More precisely, $\tau=\sqrt{\operatorname{vol}\left(\mathbb{P}^{N}(\mathbb{C})\right) /(N+1)}$. Note that the pullback in our case is nothing else as the restriction of the section to the embedded manifold $M$.

Consider the case where the coherent state embedding is an isometric (projective) Kähler embedding, i.e. the pullback of the Fubini-Study form $\omega_{F S}$ coincides with $\omega_{M}$. By Proposition 2.2 the metric $h_{L}$ in the bundle $L$ is up to a positive scalar multiple the pullback of the metric $h_{F S}$ in the hyperplane bundle: $h_{L}=\rho \cdot i^{*} h_{F S}, \rho \in \mathbb{R}, \rho>0$. Let $\epsilon_{M}$ be the Epsilon function (2.38) for the manifold $M$ and the bundle $L$. Due to the explicit description (2.41) of the Epsilon function $\epsilon_{M}$ it is up to a constant the restriction of $\epsilon_{\mathbb{P}^{N}(\mathbb{C})}$ to the embedded points. The latter is constant, hence also $\epsilon_{M}$. In more detail:

$$
\begin{aligned}
\epsilon_{M}(x)=\sum_{j=0}^{N} h_{L}\left(s_{j}, s_{j}\right)(x)=\sum_{j=0}^{N} h_{L}\left(\tau i^{*}\left(t_{j}^{\prime}\right), \tau i^{*}\left(t_{j}^{\prime}\right)\right)(x)= \\
\quad=\rho \tau^{2} \sum_{j=0}^{N} h_{H}\left(t_{j}^{\prime}, t_{j}^{\prime}\right)(i(x))=\rho \tau^{2} \epsilon_{\mathbb{P}^{N}(\mathbb{C})}(i(x))=\rho \tau^{2} \frac{N+1}{\operatorname{vol}\left(\mathbb{P}^{N}(\mathbb{C})\right)}=\rho .
\end{aligned}
$$

From this $\rho$ calculates to $\frac{N+1}{\operatorname{vol}(M)}$. 
Rawnsley calls a quantization where all the data can be obtained by pulling back the objects: bundle, forms, etc. via the (holomorphic) coherent state map from the projective space to the manifold $M$ projectively induced. Hence projectively induced quantizations have constant Epsilon functions. In fact the converse is also true:

Proposition 4.1. (Cahen, Gutt, Rawnsley, [13, p.58]) A quantization of (M, w) with quantum line bundle $(L, h)$ is projectively induced if and only if the Epsilon function is constant.

This has very interesting consequences for compact homogeneous Kähler manifolds $M \cong G / H$ with a homogeneous quantum line bundle. Recall that it is assumed in this case that the Kähler form $\omega_{M}$ and the metric in the bundle are invariant under the action of the group $G$. In particular, $\epsilon_{M}$ will be constant, hence

Corollary 4.2. For a compact homogeneous Kähler manifold which admits a homogeneous very ample quantum line bundle $L$ the coherent state embedding using this bundle is a projective Kähler embedding. In such cases the Kähler form is the pullback of the Fubini-Study form.

\subsection{Cauchy formulas.}

Let us return to the general situation of the coherent state embedding $i$, (2.34) without assuming it to be a Kählerian embedding. Fix an orthonormal basis $s_{0}, s_{1}, \ldots, s_{N}$ of the sections of the quantum line bundle $L$. Denote by $\phi$ the map from $L_{0}$ to $\mathbb{C}^{N+1}$ defined by the composition

$$
q \mapsto \overline{e_{q}} \mapsto \phi(q):=\left(\hat{q}\left(s_{0}\right), \hat{q}\left(s_{1}\right), \ldots, \hat{q}\left(s_{N}\right)\right)
$$

Clearly, $i(\pi(q))=[\phi(q)]$.

In the following, three scalar products will appear: (1) $\langle., .\rangle_{L}$, the scalar product on the space of global sections of $L$ given by $(2.21),(2)\langle., .\rangle_{\mathbb{C}^{N+1}}$, the standard scalar product on $\mathbb{C}^{N+1}$, and $(3)\langle., .\rangle_{H}$ the scalar product on the space of global sections of the hyperplane bundle $H$ on $\mathbb{P}^{N}(\mathbb{C})$. Again $(3)$ is defined by $(2.21)$, but now the manifold is $\mathbb{P}^{N}(\mathbb{C})$ and the sections are the hyperplane sections. Recall that all our scalar products are conjugate linear in the first arguments. We will call relations between these scalar products (evaluated for coherent vectors) Cauchy formulas. The first Cauchy formula is immediate from $(2.26),(4.2)$

Proposition 4.3. In the above situation we have

$$
\left\langle e_{q}, e_{q^{\prime}}\right\rangle_{L}=\left\langle\phi\left(q^{\prime}\right), \phi(q)\right\rangle_{\mathbb{C}^{N+1}} .
$$

Next we want to find relations between the scalar product of coherent vectors of $L$ over $M$ and the scalar product of coherent vectors of the hyperplane bundle $H$ over the projective space. Let $s \not \equiv 0$ be a holomorphic section of $L$, which is non-vanishing 
over the dense open subset $V_{s}$. Clearly, the scalar product of two coherent states is not defined. But, after choosing such a section we can set

$$
\left\langle e_{x}, e_{y}\right\rangle_{s}:=\left\langle e_{s(x)}, e_{s(y)}\right\rangle \text {. }
$$

If we choose another holomorphic section $s^{\prime} \not \equiv 0$ then on $V_{s} \cap V_{s^{\prime}}$ we have $s^{\prime}(x)=f(x) s(x)$ with $f$ a non-vanishing holomorphic function on this set. Hence,

$$
\left\langle e_{x}, e_{y}\right\rangle_{s^{\prime}}=\frac{1}{f(x)} \frac{1}{\overline{f(y)}}\left\langle e_{x}, e_{y}\right\rangle_{s} .
$$

Recall that Rawnsley's Epsilon function $\epsilon$ (2.38) can be written as

$$
\epsilon_{M}(x)=|s(x)|^{2}\left\langle e_{s(x)}, e_{s(x)}\right\rangle, \quad \text { where }|s(x)|^{2}:=h(s(x), s(x)) .
$$

The function

$$
\chi_{s}(x, x):=\left\langle e_{s(x)}, e_{s(x)}\right\rangle=h(s(x), s(x))^{-1} \cdot \epsilon_{M}(x)
$$

is real analytic and admits a real analytic extension to the function

$$
\chi_{s}(x, y):=\left\langle e_{x}, e_{y}\right\rangle_{s}=\left\langle e_{s(x)}, e_{s(y)}\right\rangle,
$$

which is holomorphic in $x$ and antiholomorphic in $y$.

Remark. Assume the metric $h$ in the bundle to be real-analytic. Then from (4.7) one concludes that for fixed $x$ the singularity set (in the variable $y$ ) of the extension of the metric is given by the polar divisor $\left(e_{x}\right)$.

Remark. For $\epsilon(x)=\epsilon_{M}$ a constant, we see that the scalar product of the coherent vectors is essentially given by the inverse of the local metric:

$$
\left\langle e_{x}, e_{y}\right\rangle_{s}=\frac{\epsilon_{M}}{h(s(y), s(x))} .
$$

Let us apply this to $M=\mathbb{P}^{N}(\mathbb{C})$ with the hyperplane bundle $H$ as quantum line bundle and the metric of the bundle induced by the Fubini-Study metric. Let

$V_{0}:=\left\{[z]=\left(z_{0}: z_{1}: \cdots: z_{N}\right) \mid z_{0} \neq 0\right\}$ be the standard affine chart. The points of $V_{0}$ can be given in a normalized way as $(1: w)$ with $w \in \mathbb{C}^{N}$. Take $t_{0}$ to be the section of the hyperplane bundle corresponding to the linear form $Z_{0}$, i.e. $t_{0}(w)=1$ for all $w$. We set $\hat{y}:=\left(t_{0}(y), y\right)=(1, y)$ and $\hat{x}=\left(t_{0}(x), x\right)=(1, x)$. Then

$$
h\left(t_{0}(y), t_{0}(x)\right)=\frac{1}{1+\bar{y} \cdot x}=\frac{1}{\langle\hat{y}, \hat{x}\rangle_{\mathbb{C}^{N+1}}} .
$$

Hence in this case (4.8) specializes to

$$
\left\langle e_{x}, e_{y}\right\rangle_{t_{0}}=\left\langle e_{t_{0}(x)}, e_{t_{0}(y)}\right\rangle=\langle\hat{y}, \hat{x}\rangle_{\mathbb{C}^{N+1}} \cdot F,
$$

with

$$
F:=\epsilon_{\mathbb{P}^{N}(\mathbb{C})}=\frac{N+1}{\operatorname{vol}\left(\mathbb{P}^{N}(\mathbb{C})\right)} .
$$

Now we return to the general situation. The second Cauchy formula is expressed in 
Proposition 4.4. Let $q, q^{\prime} \in L_{0}$ with $\pi(q)=x$ and $\pi\left(q^{\prime}\right)=y, i: M \rightarrow \mathbb{P}^{N}(\mathbb{C})$ the coherent state embedding (2.34), and $\phi$ the map (4.2), then on the affine chart $V_{0}$

$$
\left\langle e_{q}, e_{q^{\prime}}\right\rangle_{L}=\widehat{q}\left(s_{0}\right) \overline{\widehat{q^{\prime}}\left(s_{0}\right)} \cdot\langle\widehat{i(y)}, \widehat{i(x)}\rangle_{\mathbb{C}^{N+1}}=\frac{\widehat{q}\left(s_{0}\right) \overline{\hat{q}^{\prime}\left(s_{0}\right)}}{F} \cdot\left\langle e_{i(x)}, e_{i(y)}\right\rangle_{t_{0}} .
$$

Proof. We start from (4.3) in Proposition 4.3 and divide the vectors $\phi(q)$ and $\phi\left(q^{\prime}\right)$ on the left hand side by their first components. This can be done because we are on $V_{0}$. We obtain

$$
\left\langle e_{q}, e_{q^{\prime}}\right\rangle_{L}=\widehat{q}\left(s_{0}\right) \overline{\bar{q}^{\prime}\left(s_{0}\right)}\left\langle\phi\left(q^{\prime}\right)_{n o r m}, \phi(q)_{n o r m}\right\rangle_{\mathbb{C}^{N+1}} .
$$

Here $\phi(q)_{\text {norm }}$ is the normalized representative which has first component 1 . Using $\phi(q)_{n o r m}=i \widehat{i(\pi(q))}$ and $(4.10)$ we obtain

$$
\left\langle e_{q}, e_{q^{\prime}}\right\rangle_{L}=\widehat{q}\left(s_{0}\right) \overline{\widehat{q^{\prime}}\left(s_{0}\right)}\langle\widehat{i(y)}, \widehat{i(x)}\rangle_{\mathbb{C}^{N+1}}=\frac{\widehat{q}\left(s_{0}\right) \overline{\hat{q}^{\prime}\left(s_{0}\right)}}{F}\left\langle e_{i(x)}, e_{i(y)}\right\rangle_{t_{0}} .
$$

The third Cauchy formula is expressed in

Theorem 4.5. Let $(M, \omega)$ be a quantizable Kähler manifold with very ample quantum line bundle L. Let $i: M \rightarrow \mathbb{P}^{N}(\mathbb{C})$ be the coherent state embedding (2.34), $H$ the hyperplane section bundle. For every section $t$ of $H$ denote by $i^{*}(t)$ its pullback to $M$. Assume $t \not \equiv 0$ then over $V_{t}:=\left\{z \in \mathbb{P}^{N}(\mathbb{C}) \mid t(z) \neq 0\right\}$

$$
\left\langle e_{x}, e_{y}\right\rangle_{i^{*}(t)}=\frac{\operatorname{vol}\left(\mathbb{P}^{N}(\mathbb{C})\right)}{N+1}\left\langle e_{i(x)}, e_{i(y)}\right\rangle_{t} .
$$

Proof. First consider the section $t_{0}$. In this case $i^{*}\left(t_{0}\right)=s_{0}$. Note that in view of (4.12) and $(4.11)$ it is enough to show that $\widehat{s_{0}(x)}\left(s_{0}\right)=1$. But by definition $\widehat{s_{0}(x)}\left(s_{0}\right) \cdot s_{0}(x)=$ $s_{0}(x)$, hence

$$
\left\langle e_{x}, e_{y}\right\rangle_{s_{0}}=\left\langle e_{s_{0}(x)}, e_{s_{0}(y)}\right\rangle=\frac{1}{F}\left\langle e_{i(x)}, e_{i(y)}\right\rangle_{t_{0}} .
$$

Now take a general $t \not \equiv 0$. Recall that the complement of a zero-set of a section $(\not \equiv 0)$ is always a dense open subset. Hence the same is true for finite intersections of such sets. On the dense open set $V_{0} \cap V_{t}$ we have $t(z)=f(z) \cdot t_{0}(z)$ with $f(z)$ a holomorphic function on the intersection. For the pull-backs we obtain

$$
\left(i^{*} t\right)(x)=t(i(x))=f(i(x)) \cdot t_{0}(i(x))=\left(i^{*} f\right)(x) \cdot\left(i^{*} t_{0}\right)(x) .
$$

This implies (using (4.5))

$$
\begin{aligned}
& \left\langle e_{x}, e_{y}\right\rangle_{i^{*}(t)}=\left(\left(i^{*} f\right)(x)\right)^{-1} \cdot\left(\overline{\left(i^{*} f\right)(y)}\right)^{-1} \cdot\left\langle e_{x}, e_{y}\right\rangle_{i^{*}\left(t_{0}\right)}, \\
& \left\langle e_{i(x)}, e_{i(y)}\right\rangle_{f \cdot t_{0}}=(f(i(x)))^{-1} \cdot(\overline{f(i(y))})^{-1}\left\langle e_{i(x)}, e_{i(y)}\right\rangle_{t_{0}} .
\end{aligned}
$$

But note that $i^{*}\left(t_{0}\right)=s_{0}$ and $f(i(x))=i^{*} f(x)$. The claim follows from (4.14). 
The reason for calling the Equations (4.3), (4.12), (4.13) "Cauchy formulas" is that in the case of the Grassmannians the appearing formulas are essentially the (Binet-) Cauchy formulas [17, p.10] which give relations between the intrinsic metric on the Grassmannian and the pull-back of the Fubini-Study metric obtained via the Plücker embedding, see [2, Equations 3.13, 4.7],[3].

\subsection{Two-point functions.}

As already noted in Section 3.1 the assignment

$$
L_{0} \times L_{0} \quad \rightarrow \quad \mathbb{C}, \quad\left(q, q^{\prime}\right) \mapsto\left\langle e_{q}, e_{q^{\prime}}\right\rangle
$$

defines a real-analytic function holomorphic in $q$ and antiholomorphic in $q^{\prime}$. It can be normalized by setting

$$
\phi\left(q, q^{\prime}\right):=\frac{\left\langle e_{q}, e_{q^{\prime}}\right\rangle}{\left\|e_{q}\right\|\left\|e_{q^{\prime}}\right\|} .
$$

By the Cauchy-Schwartz inequality its absolute value is bounded by 1 .

Using (2.27) we see

$$
\phi\left(c q, c^{\prime} q^{\prime}\right)=\frac{|c|}{c} \frac{\left|c^{\prime}\right|}{\overline{c^{\prime}}} \phi\left(q, q^{\prime}\right), \quad c, c^{\prime} \in \mathbb{C}^{*} .
$$

Due to the appearing phase factors, it does not descend to a two-point function on $M$. Clearly, one way out is to take the modulus (or its square, see [14]) of (4.16). In this way one obtains the function $\psi: M \times M \rightarrow[0,1]$

$$
\psi(x, y):=\frac{\left|\left\langle e_{q}, e_{q^{\prime}}\right\rangle\right|^{2}}{\left\|e_{q}\right\|^{2}\left\|e_{q^{\prime}}\right\|^{2}}, \quad x=\pi(q), y=\pi\left(q^{\prime}\right) .
$$

This is a globally defined real-valued real-analytic function. Unfortunately, the information contained in the complex phase gets lost.

Note that the set of zeros $M(\psi)$ of (4.18) can be given with the help of polar divisors

$$
M(\psi):=\left\{(x, y) \in M \times M \mid y \in \Sigma_{x}\right\} .
$$

Recall that by Proposition 3.6 the condition is symmetric in $x$ and $y$. Clearly, the zero set $M(\phi)$ of $(4.16)$ consists of the fibers over $M(\psi)$.

Take a section $s \not \equiv 0$ of $\Gamma_{h o l}(M, L)$ and choose it as frame over $V_{s}=M \backslash(s)$. We define the function

$$
\hat{\phi}_{s}(x, y):=\frac{\left\langle e_{s(x)}, e_{s(y)}\right\rangle}{\left\|e_{s(x)}\right\|\left\|e_{s(y)}\right\|}
$$

on $V_{s} \times V_{s}$. It "represents" the two-point function (4.16). But note that $\hat{\phi}_{s}$ depends on the section $s$ which was chosen as frame.

Immediately from the definition we get

$$
\hat{\phi}_{s}(x, y)=\overline{\hat{\phi}_{s}(y, x)} \text {. }
$$


If we deal with different manifolds and if there is a danger of confusion, we will exhibit also the manifold in the notation of the two-point function (resp. of the $m$-point functions introduced later on).

Proposition 4.6. Let $i$ be the coherent state embedding (2.34). Let $t \not \equiv 0$ be a section of the hyperplane section bundle $H$ and $s=i^{*}(t)$ the corresponding section of the quantum line bundle then

(a)

$$
\hat{\phi}_{M, i^{*}(t)}(x, y)=\hat{\phi}_{\mathbb{P}^{N}(\mathbb{C}), t}(i(x), i(y))=i^{*}\left(\hat{\phi}_{\mathbb{P}^{N}(\mathbb{C}), t}\right)(x, y) .
$$

(b) For the first coordinate function $s_{0}=i^{*}\left(t_{0}\right)$ and with $\widehat{i(x)} \in \mathbb{C}^{N+1}$ the normalized homogeneous representative of $i(x)$ one has

$$
\hat{\phi}_{M, s_{0}}(x, y)=\frac{\langle\widehat{i(y)}, \widehat{i(x)}\rangle}{\|\widehat{i(x)}\|\|\widehat{i(y)}\|} .
$$

$$
\psi_{M}(x, y)=i^{*} \psi_{\mathbb{P}^{N}(\mathbb{C})}(x, y)=\psi_{\mathbb{P}^{N}(\mathbb{C})}(i(x), i(y))=\frac{\mid\langle\widehat{i(y)}, \widehat{i(x)}\rangle^{2}}{\|\widehat{i(x)}\|\left\|^{2}\right\| \widehat{i(y)} \|^{2}} .
$$

Proof. (a) is immediate from Theorem 4.5.

(b) follows from (a) using (4.10).

(c) follows from (a), resp. (b) by taking the squared modulus. Note that $\psi_{M}=$ $\hat{\phi}_{M, s} \cdot \hat{\phi}_{M, s}$ independently on the section $s$ chosen.

In the case of Perelomov's coherent states the Equation (4.23) was also called a Cauchy formula in [2, Equ. 3.13].

The two-point functions (complex-valued or real-valued) play an important rôle. From their very definition they give the transition amplitudes for coherent states. They appear as integral kernel of the Berezin transform which relates contravariant and covariant Berezin symbols, see [28]. See also the discussion in [14] for the real-valued two-point function and its relation to Calabi's diastatic function $D$. Let us add a few words on this relation. For real-analytic metrics $h$ also another two-point function $\tilde{\psi}$ is introduced in the article [14]. It is given completely in local terms of the metric. The relation $\tilde{\psi}=\exp (-D / 2)$ is shown. Certain natural behaviour under pull-backs is proven. In the case that $\epsilon=$ const (in the terminology of [14]: the bundle is regular) one obtains $\tilde{\psi}=\psi$. The key ingredients for this is equation (4.8) which relates the global scalar product with the local metric. For regular line bundles (4.24) was also proven in [14]. 


\subsection{Cyclic $m$-point functions and the three-point function.}

Let us consider the (cyclic) $m$-point function for $m \in \mathbb{N}, m \geq 2$

$$
\begin{gathered}
\Psi^{(m)}: M \times M \times \ldots \times M \rightarrow\{z \in \mathbb{C}|| z \mid \leq 1\}, \\
\Psi^{(m)}\left(x^{(1)}, x^{(2)}, \ldots, x^{(m)}\right)=\frac{\left\langle e_{q^{(1)}}, e_{q^{(2)}}\right\rangle\left\langle e_{q^{(2)}}, e_{q^{(3)}}\right\rangle \ldots\left\langle e_{q^{(m)}}, e_{q^{(1)}}\right\rangle}{\left\|e_{q^{(1)}}\right\|^{2}\left\|e_{q^{(2)}}\right\|^{2} \cdots\left\|e_{q^{(m)}}\right\|^{2}}, \\
x^{(i)}=\pi\left(q^{(i)}\right), i=1, \ldots, m .
\end{gathered}
$$

It is a complex-valued and real-analytic function in its variables. Note that the phase ambiguity of the lifts is canceled by this combination. The function $\Psi^{(m)}$ can be written in terms of the complex-valued two-point function as

$$
\Psi^{(m)}\left(x^{(1)}, x^{(2)}, \ldots, x^{(m)}\right)=\hat{\phi}_{s}\left(x^{(1)}, x^{(2)}\right) \cdot \hat{\phi}_{s}\left(x^{(2)}, x^{(3)}\right) \cdots \hat{\phi}_{s}\left(x^{(m)}, x^{(1)}\right)
$$

with respect to any section $s \not \equiv 0$ of $L$. Note that $\Psi^{(2)}=\psi$, the real-valued two-point function as defined in (4.18). But for $m>2$ the $\Psi^{(m)}$ will be complex-valued.

Proposition 4.7. Let $\Psi_{M}^{(m)}$, resp. $\Psi_{\mathbb{P}^{N}(\mathbb{C})}^{(m)}$ be the m-point function of the manifold $M$, resp. of the projective space. Let $i$ be the coherent state embedding (2.34) and $\widehat{i(x)}$ an arbitrary homogeneous representative for the point $i(x)$ then

$$
\begin{gathered}
\Psi_{M}^{(m)}\left(x^{(1)}, x^{(2)}, \ldots, x^{(m)}\right)=i^{*}\left(\Psi_{\mathbb{P}^{N}(\mathbb{C})}^{(m)}\right)\left(x^{(1)}, x^{(2)}, \ldots, x^{(m)}\right)= \\
\Psi_{\mathbb{P}^{N}(\mathbb{C})}^{(m)}\left(i\left(x^{(1)}\right), i\left(x^{(2)}\right), \ldots, i\left(x^{(m)}\right)\right)=\frac{\left\langle\widehat{i\left(x^{(2)}\right)}, \widehat{i\left(x^{(1)}\right)}\right\rangle\left\langle\widehat{i\left(x^{(3)}\right)}, \widehat{i\left(x^{(2)}\right)}\right\rangle \cdots\left\langle\widehat{i\left(x^{(1)}\right)}, \widehat{i\left(x^{(m)}\right)}\right\rangle}{\left\|\widehat{i\left(x^{(1)}\right)}\right\|\left\|^{2}\right\| \widehat{i\left(x^{(2)}\right)}\left\|^{2} \cdots\right\| \widehat{i\left(x^{(m)}\right)} \|^{2}} .
\end{gathered}
$$

The function $\Psi_{M}^{(m)}$ is invariant under cyclic permutations of its arguments.

Proof. Using (4.26) we see that from (4.22) the first equality follows. Now using (4.23) we obtain the last equality. Note that as $\widehat{i(x)}$ any homogeneous representative can be chosen (but then it has be kept fixed). The ambiguity will cancel in this combination. The invariance under cyclic permutations is clear.

We can represent the last expression in (4.27) as complex-conjugate of the similar expression where the $x^{(i)}$ appear in strictly increasing index order $\bmod m$.

Again, the zero-set of the $m$-point function can be given with the help of polar divisors.

Note that the last expression in $(4.27)$ can be rewritten as follows. Let $[u],[v] \in \mathbb{P}^{N}(\mathbb{C})$ be points with homogeneous coordinates $u, v \in \mathbb{C}^{N+1}$. The Cayley distance $d_{C}$ of the two points, i.e the geodesic distance with respect to the Fubini-Study metric is given as

$$
d_{C}([u],[v])=\arccos \frac{|\langle u, v\rangle|}{\|u\|\|v\|} .
$$


Hence the last expression in (4.27) can be given in terms of the Cayley distances of neighbouring points and an additional global phase factor $\Phi$ depending on the points.

Let us study the three-point function $\Psi=\Psi^{(3)}$ in more detail. Take $[u],[v],[w] \in$ $\mathbb{P}^{N}(\mathbb{C})$ and let $a=d_{C}([u],[v]), b=d_{C}([v],[w]), c=d_{C}([w],[u])$ be the Cayley distances. To avoid degenerate situations assume that $0<a, b, c<\pi / 2$. In particular, no point should lie in the support of the polar divisors of the other two. We can write

$$
\frac{\langle u, v\rangle\langle v, w\rangle\langle w, u\rangle}{\|u\|^{2}\|v\|^{2}\|w\|^{2}}=\cos a \cdot \cos b \cdot \cos c \cdot \mathrm{e}^{-\mathrm{i} \Phi}
$$

with the phase factor $\Phi=\Phi(u, v, w)$ defined by this formula. The phase factor is related to the shape invariant $\rho$ introduced by Blaschke and Terheggen $[10]$ for $\mathbb{P}^{2}(\mathbb{C})$, resp. by Brehm [12] for $\mathbb{P}^{N}(\mathbb{C}): \rho=\cos a \cos b \cos c \cos \Phi$.

Hangan and Masala showed that $\Phi$ has the following geometric meaning [19]: Take the (oriented) geodesic triangle $\sigma([u],[v],[w])$ with the vertices $[u],[v],[w]$, i.e. the surface swept out by the the geodesics between the point $[w]$ and all points on the geodesic between $[u]$ and $[v]$. Then

$$
\Phi=\int_{\sigma([u],[v],[w])} \omega_{\mathbb{P}^{N}(\mathbb{C})}+2 k \pi, \quad k \in \mathbb{Z} .
$$

Recall that $\omega_{\mathbb{P}^{N}(\mathbb{C})}$ is the Fubini-Study Kähler form of $\mathbb{P}^{N}(\mathbb{C})$. See also [5] for a different proof by coherent state methods. Clearly, due to the fact that $\omega_{\mathbb{P}^{N}(\mathbb{C})}$ is closed the integral does not change if we replace $\sigma([u],[v],[w])$ by any deformed surface as long as the boundary is fixed.

By applying Proposition 4.7 we see that (4.29) is the complex conjugate of the threepoint function of the projective space. We obtain

Theorem 4.8. Let $(M, \omega)$ be a quantizable Kähler manifold with very ample quantum line bundle L. Let $i: M \rightarrow \mathbb{P}^{N}(\mathbb{C})$ be the coherent state embedding (2.34). Then the three-point function

$$
\Psi^{(3)}(x, y, z)=\frac{\left\langle e_{q}, e_{q^{\prime}}\right\rangle\left\langle e_{q^{\prime}}, e_{q^{\prime \prime}}\right\rangle\left\langle e_{q^{\prime \prime}}, e_{q}\right\rangle}{\left\|e_{q}\right\|^{2}\left\|e_{q^{\prime}}\right\|^{2}\left\|e_{q^{\prime \prime}}\right\|^{2}}, \quad x=\pi(q), y=\pi\left(q^{\prime}\right), z=\pi\left(q^{\prime \prime}\right)
$$

can be written as

$$
\Psi_{M}^{(3)}(x, y, z)=\cos a \cdot \cos b \cdot \cos c \cdot \mathrm{e}^{\mathrm{i} \Phi}
$$

with $a=d_{C}(i(x), i(y)), b=d_{C}(i(y), i(z)), c=d_{C}(i(z), i(x))$ the Cayley distances in $\mathbb{P}^{N}(\mathbb{C})$ and phase

$$
\Phi=\int_{\tilde{\sigma}(i(x), i(y), i(z))} \omega_{\mathbb{P}^{N}(\mathbb{C})}
$$

where $\tilde{\sigma}(i(x), i(y), i(z))$ is any deformation of the geodesic triangle (in $\mathbb{P}^{N}(\mathbb{C})$ ) with fixed boundary given by the geodesics $\left(\right.$ in $\mathbb{P}^{N}(\mathbb{C})$ ) connecting the points $i(x), i(y)$, and $i(z)$. 
The relationship between the phase $\Phi$ of the 3-point function and the symplectic area of a geodesic triangle on the manifold itself is studied for the complex Grassmann manifolds in [5].

Acknowledgements. The authors gratefully acknowledge the Volkswagen-Stiftung for their support in the Research-in-Pairs program. We would also like to thank the Mathematisches Forschungsinstitut in Oberwolfach and its staff for the experienced hospitality. The work was finished during an invitation of S.B. at the University of Mannheim. S.B. likes to thank the DFG for supporting his stay.

\section{REFERENCES}

1. S. Berceanu, The coherent states: Old geometric methods in new quantum clothes, Preprint Institute of Atomic Physics FT-398-1994, August 1994, hep-th/9408008, 1994.

2. Coherent states and geodesics: Cut locus and conjugate locus, JGP 21 (1997), $149-168$.

3. On the geometry of complex Grassmann manifold, its noncompact dual and coherent states, Bull. Belg. Math. Soc. 4 (1997), 205-243.

4. - A remark on Berezin's quantization and cut locus, Quantizations, Deformations and Coherent States, Rep. Math. Phys (Białowieża, Poland, 1-7 July 1996) (A. Strasburger, S.T. Ali, and A. Odzijewicz, eds.), vol. 40, 1997, pp. 159-168.

5. _ Coherent states, phases and symplectic area of geodesic triangles, Talk presented at the XVII ${ }^{t h}$ workshop on geometric methods in physics, Białowieża, Poland, July 3 - 10, 1998 , math.DG/9903190, (to appear in Rep. Math. Phys).

6. Coherent states, line bundles and divisors, Quantizations, Deformations and Coherent States, Rep. Math. Phys (Białowieża, Poland, 30 June - July 6, 1997) (M. Schlichenmaier, A. Strasburger, S.T. Ali, and A. Odzijewicz, eds.), vol. 43, 1999, pp. 25-33.

7. F.A. Berezin, Quantization in complex bounded domains, Soviet Math. Dokl. 14 (1973), $1209-1213$.

8. _ Quantization, Math. USSR-Izv. 8 (1974), 1109-1165.

9. _ Quantization in complex symmetric spaces, Math. USSR-Izv. 9 (1975), 341-379.

10. W. Blaschke and H. Terheggen, Trigonometria Hermitiana, Rend. Sem. Mat. Univ. Roma 4 (1939), $153-161$.

11. M. Bordemann, E. Meinrenken, and M. Schlichenmaier, Toeplitz quantization of Kähler manifolds and $g l(n), n \rightarrow \infty$ limits, Commun. Math. Phys. 165 (1994), 281-296.

12. U. Brehm, The shape invariant of triangles and trigonometry in two-point homogeneous spaces, Geom. Dedicata 33 (1990), 50-76.

13. M. Cahen, S. Gutt, and J. Rawnsley, Quantization of Kähler manifolds I: Geometric interpretation of Berezin's quantization, JGP 7 (1990), 45-62.

14. _ Quantization of Kähler manifolds II, Trans. Amer. Math. Soc. 337 (1993), $73-98$.

15. E. Calabi, Isometric imbedding of complex manifolds, Ann. Math. 58 (1953), 1-23.

16. S.S. Chern, Complex manifolds without potential theory, Springer, New York, 1979.

17. F.R. Gantmacher, The theory of matrices, Vol. I, Chelsea Publ. Company, New York, 1990.

18. Ph. Griffiths and J. Harris, Principles of algebraic geometry, John Wiley, New York, 1978.

19. Th. Hangan and G. Masala, A geometrical interpretation of the shape invariant for geodesic triangles in complex projective spaces, Geom. Dedicata 49 (1994), 129-134.

20. R. Hartshorne, Algebraic geometry, Springer Verlag, New York, 1977.

21. S. Kobayashi and K. Nomizu, Foundations of differential geometry, Vol. I and Vol. II, Interscience Publishers, New York, 1969. 
22. B. Kostant, Quantization and unitary representations, Lectures in Modern Analysis and Applications III (C.T. Taam, ed.), Lecture Notes in Math., vol. 170, Springer, Berlin, Heidelberg, New York, 1970, pp. 87-208.

23. A. Odzijewicz, On reproducing kernels and quantization of states, Commun. Math. Phys. 114 (1988), 577-597.

24. Coherent states and geometric quantization, Commun. Math. Phys. 150 (1992), 577-597.

25. J. H. Rawnsley, Coherent states and Kähler manifolds, Quart. J. Math. Oxford Ser.(2) 28 (1977), 403-415.

26. M. Schlichenmaier, Introduction to Riemann surfaces, algebraic curves and moduli spaces, Lecture Notes in Physics, vol. 322, Springer, Berlin, Heidelberg, New York, 1990.

27. __ Berezin-Toeplitz quantization of compact Kähler manifolds, Quantization, Coherent States and Poisson Structures, Proc. XIV'th Workshop on Geometric Methods in Physics (Białowieża, Poland, 9-15 July 1995) (A. Strasburger, S.T. Ali, J.-P. Antoine, J.-P. Gazeau, and A. Odzijewicz, eds.), Polish Scientific Publisher PWN, 1998, q-alg/9601016, pp. 101-115.

28. __ Berezin-Toeplitz quantization and Berezin symbols for arbitrary compact Kähler manifolds, Talk presented at the XVII ${ }^{t h}$ workshop on geometric methods in physics, Białowieża, Poland, July 3 - 10, 1998, math.QA/9902066, 1998, (to appear in Rep. Math. Phys)..

29. _ Deformation quantization of compact Kähler manifolds by Berezin-Toeplitz quantization, Mannheimer Manuskripte Nr. 247, math.QA/9910137, 1999.

30. R.O. Wells, Differential analysis on complex manifold, Springer, Berlin, Heidelberg, New York, 1980.

31. N. Woodhouse, Geometric quantization, Clarendon Press, Oxford, 1980.

32. H. H. Wu, The equidistribution theory of holomorphic curves, Annals of Maths. Studies, vol. 164, Princeton Univ. Press, Princeton, N.J., 1970.

(Stefan Berceanu) Institute for Physics and Nuclear Engineering, Department of Theoretical Physics, PO BOX MG-6, Bucharest-Magurele, Romania

E-mail address: Berceanu@theor1.theory.nipne.ro

(Martin Schlichenmaier) Department of Mathematics and Computer Science, University of Mannheim, D7, 27, D-68131 Mannheim, Germany

E-mail address: schlichenmaier@math.uni-mannheim.de 